\title{
Neoadjuvant Therapy to Downstage the Extent of Resection of Gastrointestinal Stromal Tumors
}

\author{
Jens Jakoba, b Peter Hohenbergera \\ ${ }^{a}$ Division of Surgical Oncology and Thoracic Surgery, Department of Surgery, University Medical Center Mannheim, Medical Faculty \\ Mannheim, University of Heidelberg, Mannheim, Germany; \\ ${ }^{b}$ Department of General, Visceral and Pediatric Surgery, University Medical Center, Göttingen, Germany
}

\section{Keywords}

Preoperative treatment $\cdot$ Imatinib - Gastrointestinal stromal tumors, GIST · Resectability

\section{Summary}

Introduction: Gastrointestinal stromal tumors (GIST) are rare malignant tumors in terms of incidence, and they are not linked to specific symptoms. Often, primary tumors, particularly of the stomach, rectum, or rectovaginal space, are quite large when detected, and multivisceral resection seems to be the treatment of choice as the mainstay of therapy is complete tumor removal. If a gainof-function mutation in the KIT gene is present, drug therapy with receptor tyrosine kinase inhibitors (RTKIs) might significantly downstage primary GIST tumors. Methods: A review of the literature was performed to identify the current evidence for preoperative treatment of GIST regarding toxicity, efficacy, and oncological outcome, including mutational data from our own database. Results: Four phase II as well as several cohort studies showed acceptable toxicity and no increased perioperative morbidity of preoperative imatinib. Progressive disease during preoperative treatment was a rare event, and partial response was achieved in $40-80 \%$ of all patients. For methodological reasons, the trials cannot prove an oncological long-term superiority of preoperative treatment. Conclusion: Preoperative therapy with imatinib is safe and recommended for patients with locally advanced GIST. Neoadjuvant imatinib therapy may enable less invasive and organ-sparing surgery, avoid tumor rupture during extensive resectional procedures, and improve the quality of perioperative RTKI treatment.

(C) 2018 S. Karger GmbH, Freiburg

\section{Introduction}

Gastrointestinal stromal tumors (GIST) are rare cancers with an age-adjusted incidence of 1.5 per 100,000 inhabitants. Nevertheless, GIST are the most common mesenchymal tumors of the gastrointestinal tract [1]. Surgical removal is the most important therapy for non-metastatic tumors, and $\mathrm{R} 0$ resection remains the only curative option and cornerstone of GIST treatment [2, 3]. Nevertheless, up to $50 \%$ of all patients with primarily non-metastatic GIST develop tumor recurrences depending on size, proliferative activity, localization of the primary, and intraoperative tumor rupture [4]. Frequently, local recurrences as well as peritoneal or hepatic metastases are not accessible for surgical treatment despite concurrent receptor tyrosine kinase inhibitor (RTKI) treatment [5].

Progress in understanding the underlying molecular mechanisms of GIST has led to the development of rational, targeted therapy approaches. Approximately $80 \%$ of GIST tumors have a gain-of-function mutation of the c-kit or PDGF receptor [6-8]. Targeted therapy with the small-molecule RTKI imatinib has led to a dramatic improvement of the prognosis of patients with metastatic GIST [9-11]. The European Medicines Agency and the Food and Drug Administration did not only approve imatinib for the treatment of metastatic disease but also as an adjuvant therapy for patients with a significant risk of tumor recurrence $[12,13]$. In sensitive tumors, imatinib often results in a partial response and has been proposed as preoperative treatment very early. The options of neoadjuvant imatinib therapy are: less invasive and organ-sparing surgery, avoidance of tumor rupture during multivisceral resection of untreated primary tumors, and improved basis for adjuvant RTKI treatment. This review summarizes the current evidence of neoadjuvant treatment of GIST.

\section{KARGER}

() 2018 S. Karger GmbH, Freiburg
PD Dr. med. Jens Jakob

Division of Surgical Oncology and Thoracic Surgery, Department of Surgery

University Medical Center Mannheim, Medical Faculty Mannheim, University of Heidelberg

Theodor-Kutzer-Ufer 1-3, 68163 Mannheim, Germany

jjakob@gmx.org 


\section{Principles of Surgery and Different Rationales for Preoperative Treatment in Locally Advanced GIST}

Both the US National Comprehensive Cancer Network (NCCN) and the European Society for Medical Oncology (ESMO) guidelines describe the R0 resection of GIST as the main goal of surgical therapy $[2,3]$. Although individual studies imply an advantage in terms of local recurrence-free survival for resection margins larger than $1 \mathrm{~cm}$, marginal resections are considered appropriate as long as microscopically tumor-free margins are established $[14,15]$. In contrast to gastrointestinal carcinomas, systematic lymphadenectomy is not indicated in GIST since lymph node metastases are a rare event. Because of both principles, complete tumor resections along with atypical or segmental removal of the stomach, the small intestine, or the rectum are regarded as oncologically adequate. Whereas in rectal cancer anterior rectal resection with total mesorectal excision is indicated, a marginal transanal full-thickness R0 resection may be sufficient for a rectal GIST of the similar localization and diameter [16].

Both guidelines mentioned above clearly recommend the use of neoadjuvant therapy to treat locally advanced GIST if surgical morbidity and mutilating surgery may be avoided. The US guideline mentions rectal GIST as an exemplary indication for neoadjuvant therapy [2]. If downsizing of the tumor enables sphincter-preserving surgery, neoadjuvant imatinib should be considered. Performing a transanal resection without opening the peritoneum might even have the potential to decrease the risk of peritoneal tumor spread, which are most likely the sequelae of intraoperative contamination or direct invasion by the tumor. Transanal local excision leaves the peritoneal cavity intact and may thus reduce the rate of peritoneal metastases [16]. Whereas the latter is a theoretical advantage, sphincter preservation in rectal GIST after preoperative downsizing has been clearly demonstrated in a number of case series [17]. In case of gastric GIST, located in the cardia, local excision without resection of the esophagogastric junction may be achieved (fig. 1); in case of duodenal GIST, a wedge resection may replace a Whipple's procedure [18-21].

Organ-sparing surgery in gastric GIST impacts adjuvant imatinib therapy. Evaluation of imatinib blood levels in patients under treatment demonstrated that patients after total gastrectomy had lower drug levels than those with partial gastric resection only [22]. Postoperative imatinib treatment is indicated for a large proportion of patients with advanced gastric GIST [2,3]. Therefore, minimizing the extent of removal of the stomach for gastric GIST primary tumors will not only be advantageous in the setting of adjuvant treatment but will also positively influence drug therapy for metastatic disease. Translational studies evaluated the relationship between imatinib blood levels and treatment response. It revealed that plasma levels below $760 \mathrm{ng} / \mathrm{l}$ after 3 months of treatment are associated with a worse prognosis [23]. Interestingly, in this cohort analyzed in France, there was also a strong correlation between the extent of gastric resection and imatinib blood levels. This indicates that preoperative treatment with imatinib in patients with upper gastrointestinal tract GIST results in higher drug levels both pre-

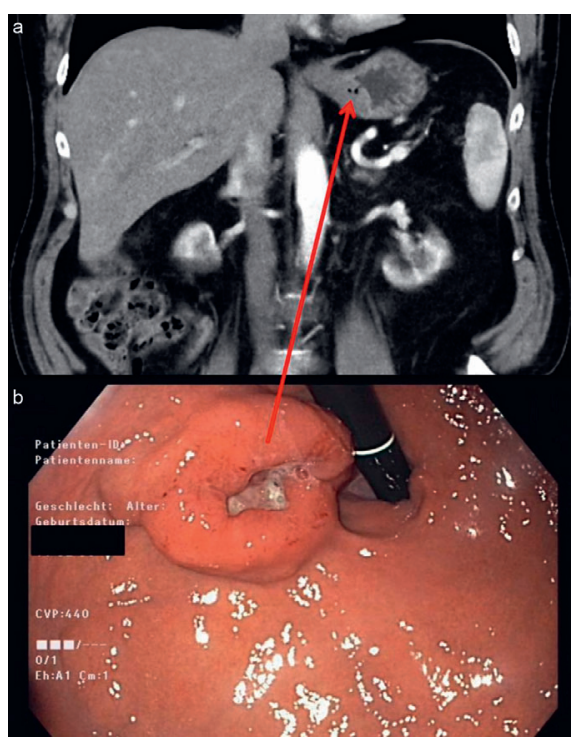

Fig. 1. a Endoscopy and $\mathbf{b}$ computed tomography of a patient with an ulcerated intermediate-risk gastric gastrointestinal stromal tumor at the esophagogastric junction harboring an exon 11 mutation of c-kit. 12 months of preoperative treatment with imatinib resulted in significant tumor shrinkage. Consequently, a local pR0 tumor resection with margin of clearance of less than $1 \mathrm{~cm}$ was performed, thus avoiding resection of the esophagogastric junction. Postoperatively, the patient is currently free from recurrence for 52 months.

and postoperatively: preoperatively by temporarily leaving the stomach in place and postoperatively by limiting the extent of gastric resection. This may result in an overall improvement of the prognosis of patients with upper gastrointestinal GIST tumors (fig. 1).

Tumor rupture is a major risk factor in GIST. Cohort studies show a recurrence rate of close to $100 \%$ after spontaneous or intraoperative tumor rupture [4, 24-27]. Postoperative RTKI therapy may delay tumor relapse after rupture - and must not be interrupted - but it cannot prevent local or peritoneal recurrence. Since peritoneal GIST metastases have a worse prognosis compared to those of the liver, any attempt should be made to decrease the risk of intraoperative tumor rupture [28]. There are no prospective studies on this subject; however, there is strong belief that downsizing and devitalization of large, fragile GIST by preoperative treatment improves surgical handling and avoids tumor rupture (fig. 2).

Surgery for GIST may be performed using conventional, laparoscopic, or robotic techniques $[29,30]$. No randomized trials have been performed to compare surgical techniques. Reports from single-center cohorts are strongly biased since they are usually reported by experienced laparoscopic surgeons who would only perform open surgery in the most complex cases. In principle, minimally invasive techniques are ascribed lower postoperative morbidity and better cosmetic results. Consequently, downsizing of a GIST by preoperative treatment may go along with the advantages of minimally invasive surgery in general. Nevertheless, surgeons need to bear in mind that the guidelines discourage to perform minimally invasive surgery if there is any risk to increase the rate of 


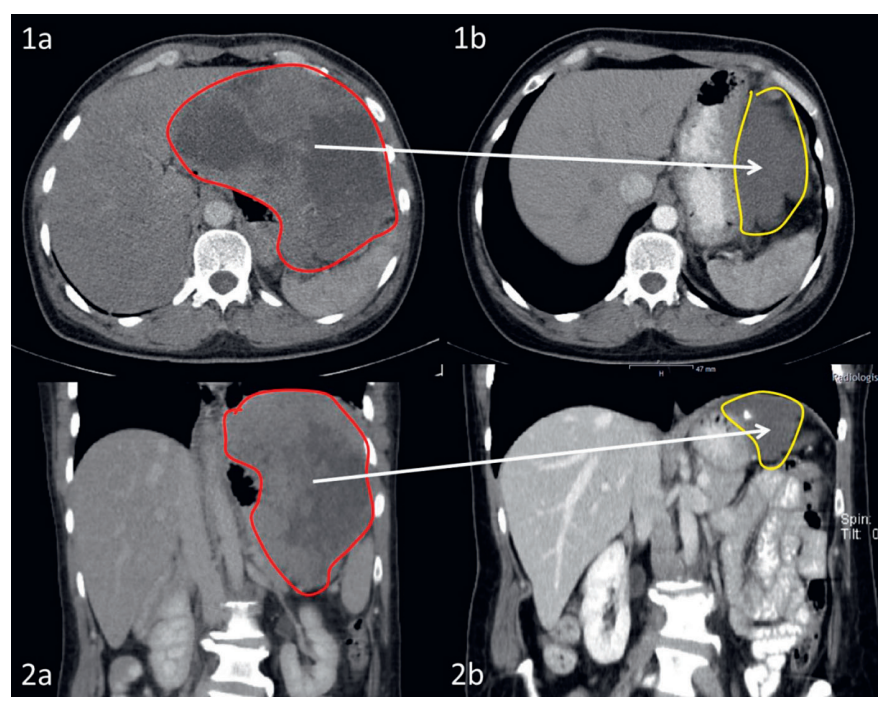

Fig. 2. Partial response documented on axial ( $\mathbf{1} \mathbf{a}$ and $\mathbf{2 a})$ and coronary (2a and $\mathbf{2} \mathbf{b}$ ) computed tomography imaging of a high-risk gastric gastrointestinal stromal tumor harboring an exon 11 mutation of c-kit before (1a and $\mathbf{1} \mathbf{b}$ ) and after ( $2 \mathbf{a}$ and $\mathbf{2} \mathbf{b}$ ) induced by preoperative treatment with imatinib for 15 months. As a result, the patient underwent wedge resection of the stomach instead of total gastrectomy plus splenectomy. Currently, the patient receives adjuvant imatinib in the second year after surgery and remains recurrence-free.

tumor rupture, although this notion lacks evidence from data proving that minimally invasive surgery poses the patients at a higher risk for tumor fragmentation [3, 31]. Beside faster recovery after less invasive surgery, tumor response may also lead to less intraoperative bleeding in otherwise highly vascularized large GIST tumors through tumor devitalization. Monitoring of neoadjuvant therapy by imaging studies demonstrates that neovascularization decreases significantly [32]. In summary, the opportunities of preoperative imatinib therapy may be less invasive and organ-sparing surgery with avoidance of tumor rupture and bleeding as well as improved quality of perioperative RTKI treatment.

\section{Neoadjuvant Treatment in Case of GIST Presenting as an Emergency}

The question is whether the treatment policy has to be changed in patients being diagnosed with upper gastrointestinal bleeding in locally advanced gastric or duodenal GIST in comparison to those patients presenting in an elective state. More than $10 \%$ of the patients with stomach GIST and almost $15 \%$ of those with GIST of the duodenum present as an emergency, mainly with upper gastrointestinal bleeding [33]. There are no prospective studies but a lot of anecdotal publications reporting acute bleeding rates of up to $55 \%$ and recommending sleeve gastrectomy as well as successful control of bleeding by imatinib with subsequent tumor resection after downsizing [34-36]. Our institutional policy would always intend to treat a primary stomach GIST, which becomes symptomatic with an upper gastrointestinal bleeding, by a neoadjuvant therapy with imatinib, if there is a sensitive mutation. The immediate suspension of tumor proliferation and the rapid reduction of tumor perfusion (detectable in perfusion magnetic resonance imaging (MRI), contrast-enhanced computed tomography (CECT), and positron emission tomography (PET)) is almost always expected to stop the bleeding. The prerequisite is that an imatinibsensitive mutation is present (fig. 3). We analyzed the mutational spectrum of all GIST in our database of the past 15 years and compared the data to those tumors in which we would look for a downsizing by neoadjuvant therapy, typically with imatinib. Of 516 GIST primary tumors, 407 (78.8\%) had an imatinib-sensitive exon 11 mutation. The subgroup of gastric GIST larger than $10 \mathrm{~cm}$, duodenal or rectal GIST larger than $6 \mathrm{~cm}$, and esophageal GIST larger than $5 \mathrm{~cm}$ comprised of 167 tumors. In this group, 118 (70.6\%) patients presented with an exon 11 mutation (n.s.). Interestingly, if one looks at exon 9 mutations, the overall group consists of 48/516 patients $(9.3 \%)$, whereas the group with larger tumors and potential neoadjuvant indication only showed $5 / 167$ patients (3\%) with this subtype requiring $800 \mathrm{mg}$ of imatinib to respond. Since it often takes a few days before the mutational data are available, we would immediately start therapy with imatinib if we were to detect a spindle cell tumor with upper gastrointestinal bleeding which otherwise has the characteristic signs of GIST. The rate of side effects is low, and the probability to respond to therapy is high.

\section{Review of the Literature}

RTOG 0132 was one of the first prospective studies to test the feasibility of preoperative imatinib [37]. The study population included both primary non-metastatic as well as recurrent and (oligo-)metastatic patients. All tumor lesions had to be resectable. Patients received $600 \mathrm{mg}$ imatinib daily for 8-12 weeks preoperatively and for 2 years postoperatively. 62 patients were included, of whom 53 patients were evaluated, including 30 patients with primary non-metastatic GIST (table 1). As in the other studies, primary tumors of the duodenum and rectum were significantly overrepresented compared to the total population of GIST patients. This indicates the preference of preoperative treatment for tumors at difficult surgical locations. The median tumor size was $9 \mathrm{~cm}$. The short duration of the preoperative therapy has been chosen because the registration studies for imatinib had shown treatment response in a number of cases already after 10 weeks. This explains the rather lower rate of partial remissions compared to the other phase II trials (table 1). Fortunately, none of the patients showed tumor progression during preoperative therapy. Treatment was regarded to be safe in oncological terms. The wound infection rate at surgery was $7 \%$; one anastomotic leakage, one bile leakage, and two intra-abdominal abscesses occurred in 45 documented surgical procedures.

NCT 00290485 was a Canadian prospective phase II study [38]. Between 2004 and 2007, 14 patients with primary non-metastatic GIST were included. The therapeutic intervention consisted of the preoperative administration of $400 \mathrm{mg}$ imatinib for up to 12 months. The authors of the study envisaged the first imaging after 
Fig. 3. Comparison of mutational pattern comparing gastrointestinal stromal tumor (GIST) primary tumors (Mannheim Registry, 2004-2017, n = 822). Left side: Distribution in the overall group with mutational data available irrespective of tumor size $(\mathrm{n}=516)$. Right side: Subgroup of locally advanced GIST in which neoadjuvant treatment could be considered, i.e. esophageal GIST $>5 \mathrm{~cm}$, gastric GIST $>10 \mathrm{~cm}$, and duodenal $/$ rectal GIST $>6 \mathrm{~cm}$.

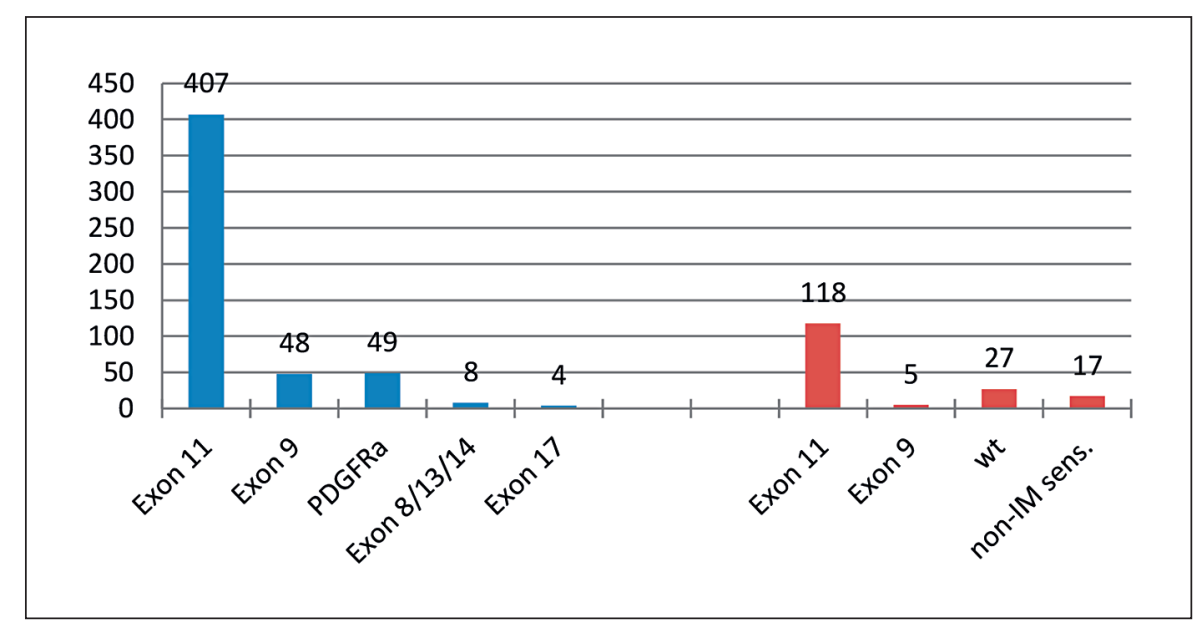

Table 1. Prospective trials of preoperative imatinib for locally advanced gastrointestinal stromal tumors

\begin{tabular}{|c|c|c|c|c|c|c|c|c|}
\hline Trial & Design & $\begin{array}{l}\text { Treatment } \\
\text { regimen }\end{array}$ & $\begin{array}{l}\text { Patients, } \\
\mathrm{n}\end{array}$ & $\begin{array}{l}\text { Progressive dis- } \\
\text { ease }\end{array}$ & $\begin{array}{l}\text { Partial } \\
\text { response, \% }\end{array}$ & $\begin{array}{l}\text { Surgery, } \\
\mathrm{n}\end{array}$ & $\begin{array}{l}\mathrm{R} 0 \\
\text { resections, } \mathrm{n}\end{array}$ & $\begin{array}{l}\text { Progression-free } \\
\text { survival }\end{array}$ \\
\hline Eisenberg, 2009 [37] & phase II & $\begin{array}{l}600 \mathrm{mg} \text { imatinib for } \\
8-12 \text { weeks }\end{array}$ & $30^{\mathrm{a}}$ & none & 7 & 26 & 20 & $83 \%$ at 2 years \\
\hline Doyon, 2012 [38] & phase II & $\begin{array}{l}400-600 \mathrm{mg} \text { imatinib } \\
\text { for up to } 12 \text { months }\end{array}$ & 14 & none & 43 & 11 & 11 & $64 \%$ at 4 years \\
\hline Hohenberger, $2012^{\mathrm{b}}[40]$ & phase II & $\begin{array}{l}400 \mathrm{mg} \text { imatinib up to } \\
12 \text { months }\end{array}$ & 41 & 2 & 82 & 40 & 33 & $85 \%$ at 3 years \\
\hline Kurokawa, 2017 [39] & phase II & $\begin{array}{l}400 \mathrm{mg} \text { imatinib for up } \\
\text { to } 9 \text { months }\end{array}$ & 53 & none & 62 & 50 & 48 & $89 \%$ at 2 years \\
\hline
\end{tabular}

${ }^{a}$ Only non-metastatic patients.

b26 patients had less extensive surgery after preoperative treatment compared to the initially planned procedure.

9 weeks to enable early tumor resection in the case of progressive disease. Imatinib had a favorable toxicity profile. Skin toxicity and nausea were the most common side effects. 3 patients had a tumor hemorrhage and 1 patient underwent surgery before completion of preoperative treatment under suspicion of tumor progression due to aggravating symptoms. However, this patient did not show tumor perforation or tumor progression intraoperatively. Again, none of the patients showed tumor progression during preoperative treatment, and $62 \%$ developed a partial remission according to RECIST. 1 patient with an esophagogastric resection suffered from an anastomosis leakage postoperatively.

Primary endpoint of the Japanese-Korean UMIN00000003114 study was the R0 resection rate of gastric GIST larger than $10 \mathrm{~cm}$ [39]. GIST of other localizations were not included in this trial. Study intervention was the administration of $400 \mathrm{mg}$ imatinib for 6-9 months. 56 patients were included in the trial, 53 of whom were eligible for evaluation. None of the patients developed tumor progression. A partial remission was achieved in $62 \%$ of patients. The most common grade III side effects of preoperative imatinib were rash $(9 \%)$ and neutropenia $(8 \%)$. In the cohort $(n=53)$ undergoing surgery only 3 patients required total gastrectomy for complete tumor removal. The R0 resection rate was $96 \% .2$ patients suffered from anastomotic leakage, 2 patients developed wound infections, and 1 patient suffered from abdominal abscess formation.

The German Apollon Trial (NCT00112632) was a prospective phase II study in locally advanced GIST to test the efficacy of preoperative imatinib $400 \mathrm{mg}$ for 6 months [40]. 46 patients were included into the trial. Dose reductions and therapy interruptions became necessary in 2 patients. In another 2 patients, early tumor resection was performed due to tumor progression. The median tumor size of all other patients decreased from 9 to $5 \mathrm{~cm}$ during preoperative treatment. The most important finding of the Apollon trial was that 26 patients had less extensive surgery after preoperative treatment compared to the initially planned procedure; in $70 \%$ of all patients who were scheduled for full-organ resection (e.g. total gastrectomy or abdominoperineal/low anterior resection), a limited organ resection could be performed. The R0 resection rate was $88 \%$. Perioperative morbidity was not at all increased compared to similar resections for epithelial cancer.

Similar results were reported by a smaller prospective Swedish 
trial. 10 high-risk GIST patients received $400 \mathrm{mg}$ imatinib preoperatively for up to 12 months [21]. The median tumor size decreased from 20 to $10 \mathrm{~cm}$. In 8 out of 10 patients, a relevant reduction in the extent of tumor resection was achieved (e.g. wedge resection of the stomach instead of total gastrectomy).

The analysis of the pooled databases from 10 experienced centers of the EORTC Soft Tissue and Bone Sarcoma Group represents the largest published cohort of GIST patients treated by preoperative imatinib and tumor resection [41]. With a median follow-up of 46 months, 161 patients with GIST of the stomach (55\%), rectum (20\%), and duodenum (10\%) had an R0 resection rate of $83 \%$ and a 5 -year progression-free survival (PFS) rate of $65 \%$. Treatment toxicity corresponded to the known side effects of imatinib when administered in metastatic disease. Obviously, preoperative therapy was particularly indicated for GIST at difficult anatomical locations such as the rectum or duodenum since both are clearly overrepresented in relation to their incidence.

\section{Preoperative Treatment of Locally Advanced GIST}

\section{Drug Selection}

Imatinib is an orally administered, well-tolerated and effective RTKI, which is approved for first-line treatment of unresectable and metastatic GIST and for adjuvant treatment in tumors with a relevant risk for recurrence. More than $80 \%$ of primary tumors harbor mutations in the KIT or PDGFRA proto-oncogene $[6,8]$. Several phase II trials have demonstrated the safety and efficacy of preoperative imatinib, and if an imatinib-sensitive mutation is present, the drug is the first choice for neoadjuvant treatment. The standard dose of imatinib is $400 \mathrm{mg}$ given daily per os. In case of mutations in the exon 9 of KIT, the dose must be increased to 800 $\mathrm{mg}$ to achieve a similar response as in exon 11 mutations [42].

Other receptor tyrosine kinases such as sunitinib and regorafenib are approved for imatinib-resistant tumors and secondor third-line therapy $[43,44]$. An institutional study by Raut et al. [45] evaluated surgery for metastatic GIST in 50 patients after sunitinib therapy. Their conclusion was that the combination is feasible; however, the complication rates were significantly higher than after imatinib pretreatment. The risk of postoperative wound (and anastomotic) healing disorders may occur due to the anti-angiogenic properties, probably related to the inhibition of angiogenesis by sunitinib and regorafenib. Regarding the increased toxicity of both drugs (e.g. hypertension and hand-foot syndrome), both drugs do not seem to be good candidates for perioperative use over several months. Surgeons should bear in mind that both drugs should be stopped 2 weeks prior to any abdominal surgery to ensure proper wound (and anastomotic) healing [46].

Nilotinib is a selective RTKI that has a similar activity against KIT and PDGFR as imatinib. The oncological efficacy of nilotinib as a first-line treatment has been compared to that of imatinib in a randomized phase III trial [47]. The primary endpoint of the trial was PFS. PFS was better with imatinib (hazard ratio (HR) 1.47) for all patients and especially in patients with KIT exon 9 mutations
(HR 32.46) but similar between arms in the KIT exon 11 mutation subgroups (HR 1.12). The overall response rate was also similar to that of imatinib (40 vs. 50\%). The most frequent adverse events of nilotinib were rash, nausea, abdominal pain, and fatigue. Thus, nilotinib could be an alternative drug in the neoadjuvant setting in patients intolerant of imatinib who suffer from a GIST harboring a KIT exon 11 mutation.

\section{Pretreatment Biopsies and Mutational Analysis}

The prerequisite of preoperative treatment is the histological proof of the diagnosis of GIST and its mutational status. Although gastric and rectal GIST are easily assessable by endoscopy, often endoluminal biopsy does not prove the diagnosis and percutaneous core needle biopsies could be required. Any biopsy of GIST through the abdominal wall has in principal a risk to cause tumor cell contamination of the abdominal cavity or wall, though. Current biopsy techniques include image guidance and the use of coaxial needles to minimize this risk. A systematic evaluation of the SSG XVIII/AIO trial comparing 1 and 3 years of adjuvant imatinib in high-risk GIST was performed to determine the rate of biopsyassociated recurrences [48]. Analysis of 397 patients of whom 47 underwent percutaneous biopsy revealed that there was no difference in the recurrence rates between the two groups. The authors concluded that preoperative diagnostic percutaneous biopsy of a suspected GIST does not increase the risk for GIST recurrence in patients who receive adjuvant imatinib after the biopsy.

Imatinib is active in GIST with mutations in the exon 11 and 9 of KIT gene and non-D842V PDGFRA mutations which comprise more than $80 \%$ of all primary tumors [8]. Imatinib is not active in exon 13, 14 and 17 mutations of KIT, in D842V PDGFR mutations, and in BRAF-mutated GIST, which comprise another $15 \%$ of all tumors. In succinate dehydrogenase-deficient GIST, some activity of imatinib is seen [49]. Although all reported phase II trials of preoperative treatment required pretreatment biopsy, they did not include mutational analysis as a prerequisite for neoadjuvant treatment. Nevertheless, only few patients developed tumor progression and none of the authors reported cases of irresectability because of imatinib resistance. Nowadays, genetic testing of GIST biopsies is a standard of care and is usually available within days or weeks. If test results are inconclusive, we begin with preoperative imatinib, repeat mutational analysis, and shorten the follow-up interval in order not to miss tumor progression.

\section{Duration and Monitoring of Preoperative Treatment}

Compared to the other phase II studies, the design of the RTOG 0132 study provided the shortest preoperative therapy time of 8-12 weeks [37]. The response rate according to RECIST of the RTOG 0132 study was only $7 \%$. Controlled trials with a longer therapy duration of 6-12 months showed response rates of more than $50 \%$; the response rate in the cohort study of the EORTC centers was $80 \%$ [41]. The authors of the EORTC 62005 trial, evaluating PFS of locally advanced and metastatic GIST with 400 versus $800 \mathrm{mg}$ imatinib, reported a median time to achieve a partial response according to RECIST at almost 9 months [50]. A dedi- 
cated evaluation of imaging studies performed during preoperative therapy by the Boston group showed the best response after 16 weeks [51]. Thus, we can assume a minimum therapy interval of 4 months. None of the authors of neoadjuvant studies described patients with progressive disease after initial tumor response during preoperative therapy - provided the therapy was not continued for more than 12 months or resection was refused by the patient [52]. A preoperative treatment duration of 6-9 months seems reasonable until the point when no further reduction of tumor size can be expected. The minimum therapy duration should be 4 months to achieve treatment response. The maximum duration should not exceed 12 months to avoid tumor progression after initial response.

Adjuvant imatinib is recommended in those patients with a relevant risk of tumor recurrence and whose tumors bear imatinibsensitive mutations $[2,3]$. None of the published neoadjuvant trials evaluated whether adjuvant treatment may be omitted after preoperative imatinib administration. We therefore recommend administering adjuvant treatment according to the ESMO or NCCN guidelines.

The most experience in response assessment for GIST exists for CT [32]. CT is widely available and can be carried out quickly, safely, and cost-effectively. Response assessment by CT is reliable, and a second radiologist may review imaging if necessary. Therefore, we generally recommend abdominal CECT at 3-month intervals during preoperative treatment. MRI may be indicated for planning surgical procedures, e.g. in rectum GIST. PET-CT shows a metabolic tumor response within the first 2 weeks of therapy. PET-CT can therefore be indicated, particularly in cases of unclear mutation status, high toxicity, or clinical signs of progress.

\section{Conclusion}

The mainstay of GIST therapy is complete resection. Due to a typical gain-of-function mutation in the KIT or PDGFRA gene, preoperative treatment with imatinib is a rationale to achieve tumor devitalization and downsizing in locally advanced GIST. Response to treatment frequently enables less invasive and organsparing surgery and may help to avoid tumor rupture. Since RTKI drug uptake correlates inversely with the extent of gastric resection, preoperative treatment and less invasive surgery may improve the quality of adjuvant RTKI treatment in upper gastrointestinal GIST. Pretreatment biopsy and mutational analysis of KIT and PDGFRA are mandatory. If the mutation is sensitive to the drug, re-staging intervals do not need to be too close. The drug of choice is imatinib; preoperative treatment is safe and effective if given typically for 6-12 months.

\section{Disclosure Statement}

No conflict of interest.

\section{References}

1 Ressing M, Wardelmann E, Hohenberger $\mathrm{P}$, et al: Strengthening health data on a rare and heterogeneous disease: sarcoma incidence and histological subtypes in Germany. BMC Public Health 2018;18:235

2 Network NNCC. NCCN Clinical Practice Guidelines in Oncology (NCCN Guidelines) Soft Tissue Sarcoma Version 2.2018 - March 27, 2018. https://www.nccn org/professionals/physician_gls/pdf/sarcoma.pdf (accessed 10.06.2018)

$\checkmark 3$ Casali PG, Abecassis N, Bauer S, et al.; ESMO Guidelines Committee and EURACAN: Gastrointestinal stromal tumours: ESMO-EURACAN Clinical Practice Guidelines for diagnosis, treatment and follow-up. Ann Oncol 2018;DOI: 10.1093/annonc/mdy095.

4 Joensuu H, Vehtari A, Riihimaki J, et al: Risk of recurrence of gastrointestinal stromal tumour after surgery: an analysis of pooled population-based cohorts. Lancet Oncol 2012;13:265-274

5 Mussi C, Ronellenfitsch U, Jakob J, et al: Post-imatinib surgery in advanced/metastatic GIST: is it worthwhile in all patients? Ann Oncol 2010;21:403-408.

6 Wozniak A, Rutkowski P, Schoffski P, et al: Tumor genotype is an independent prognostic factor in primary gastrointestinal stromal tumors of gastric origin: a European multicenter analysis based on ConticaGIST. Clin Cancer Res 2014;20:6105-6116.
Joensuu H, Wardelmann E, Sihto H, et al: Effect of KIT and PDGFRA mutations on survival in patients with gastrointestinal stromal tumors treated with adjuvant imatinib: an exploratory analysis of a randomized clinical trial. JAMA Oncol 2017;3:602-609.

8 Huss S, Pasternack H, Ihle MA, et al: Clinicopathologi$\mathrm{cal}$ and molecular features of a large cohort of gastrointestinal stromal tumors (GISTs) and review of the literature: BRAF mutations in KIT/PDGFRA wild-type GISTs are rare events. Hum Pathol 2017;62:206-214.

9 Casali PG, Zalcberg J, Le Cesne A, et al: Ten-year progression-free and overall survival in patients with unresectable or metastatic GI stromal tumors: long-term analysis of the European Organisation for Research and Treatment of Cancer, Italian Sarcoma Group, and Australasian Gastrointestinal Trials Group Intergroup phase III randomized trial on imatinib at two dose levels. J Clin Oncol 2017;35:1713-1720.

10 Patrikidou A, Domont J, Chabaud S, et al: Long-term outcome of molecular subgroups of GIST patients treated with standard-dose imatinib in the BFR14 trial of the French Sarcoma Group. Eur J Cancer 2016;52: $173-180$.

11 Blanke CD, Demetri GD, von Mehren M, et al: Longterm results from a randomized phase II trial of standard- versus higher-dose imatinib mesylate for patients with unresectable or metastatic gastrointestinal stromal tumors expressing KIT. J Clin Oncol 2008;26: $620-625$.
12 Joensuu H, Eriksson M, Sundby Hall K, et al: One vs three years of adjuvant imatinib for operable gastrointestinal stromal tumor: a randomized trial. JAMA 2012;307:1265-1272.

13 Dematteo RP, Ballman KV, Antonescu CR, et al: Adjuvant imatinib mesylate after resection of localised, primary gastrointestinal stromal tumour: a randomised, double-blind, placebo-controlled trial. Lancet 2009; 373:1097-1104.

14 Zhi X, Jiang B, Yu J, et al: Prognostic role of microscopically positive margins for primary gastrointestinal stromal tumors: a systematic review and meta-analysis. Sci Rep 2016;6:21541.

15 Ahlen J, Karlsson F, Wejde J, Nilsson IL, Larsson C, Branstrom R: Wide surgical margin improves the outcome for patients with gastrointestinal stromal tumors (GISTs). World J Surg 2018;42:2512-2521.

16 Jakob J, Mussi C, Ronellenfitsch U, et al: Gastrointestinal stromal tumor of the rectum: results of surgical and multimodality therapy in the era of imatinib. Ann Surg Oncol 2013;20:586-592.

17 Wilkinson MJ, Fitzgerald JE, Strauss DC, et al: Surgical treatment of gastrointestinal stromal tumour of the rectum in the era of imatinib. Br J Surg 2015;102:965971

18 Tielen R, Verhoef C, van Coevorden F, et al: Surgical treatment of locally advanced, non-metastatic, gastrointestinal stromal tumours after treatment with imatinib. Eur J Surg Oncol 2013;39:150-155. 
19 Colombo C, Ronellenfitsch U, Yuxin Z, et al: Clinical, pathological and surgical characteristics of duodenal gastrointestinal stromal tumor and their influence on survival: a multi-center study. Ann Surg Oncol 2012 19:3361-3367.

20 Stiekema J, Kol S, Cats A, Yazdi AT, van Coevorden F, van Sandick JW: Surgical treatment of gastrointestinal stromal tumors located in the stomach in the imatinib era. Am J Clin Oncol 2015;38:502-507.

-21 Sjolund K, Andersson A, Nilsson E, Nilsson O, Ahlman $\mathrm{H}$, Nilsson B: Downsizing treatment with tyrosine kinase inhibitors in patients with advanced gastrointestinal stromal tumors improved resectability. World J Surg 2010;34:2090-2097.

22 Yoo C, Ryu MH, Kang BW, et al: Cross-sectional study of imatinib plasma trough levels in patients with advanced gastrointestinal stromal tumors: impact of gastrointestinal resection on exposure to imatinib. J Clin Oncol 2010;28:1554-1559.

23 Bouchet S, Poulette S, Titier K, et al: Relationship between imatinib trough concentration and outcomes in the treatment of advanced gastrointestinal stromal tumours in a real-life setting. Eur J Cancer 2016;57:3138.

24 Hohenberger P, Ronellenfitsch U, Oladeji O, et al: Pattern of recurrence in patients with ruptured primary gastrointestinal stromal tumour. Br J Surg 2010;97: 1854-1859.

25 Nishida T, Cho H, Hirota S, Masuzawa T, Chiguchi G, Tsujinaka T: Clinicopathological features and prognosis of primary GISTs with tumor rupture in the real world. Ann Surg Oncol 2018;25:1961-1969.

26 Joensuu H, Eriksson M, Hall KS, et al: Risk factors for gastrointestinal stromal tumor recurrence in patients treated with adjuvant imatinib. Cancer 2014;120:23252333.

27 Holmebakk T, Bjerkehagen B, Boye K, Bruland O, Stoldt S, Sundby Hall K: Definition and clinical significance of tumour rupture in gastrointestinal stromal tumours of the small intestine. Br J Surg 2016;103: 684-691.

28 Bauer S, Rutkowski P, Hohenberger P, et al: Long-term follow-up of patients with GIST undergoing metastasectomy in the era of imatinib - analysis of prognostic factors (EORTC-STBSG collaborative study). Eur J Surg Oncol 2014;40:412-419.

29 Vicente E, Quijano Y, Ielpo B, et al: Robot-assisted resection of gastrointestinal stromal tumors (GIST): a single center case series and literature review. Int J Med Robot 2016;12:718-723.

-30 De Vogelaere K, Hoorens A, Haentjens P, Delvaux G: Laparoscopic versus open resection of gastrointestinal stromal tumors of the stomach. Surg Endosc 2013;27: 1546-1554.
31 Ronellenfitsch U, Staiger W, Kähler G, Strobel P, Schwarzbach M, Hohenberger P: Perioperative and oncological outcome of laparoscopic resection of gastrointestinal stromal tumour (GIST) of the stomach. Diagn Ther Endosc 2009;2009:286138.

32 Dimitrakopoulou-Strauss A, Ronellenfitsch U, Cheng $\mathrm{C}$, et al: Imaging therapy response of gastrointestinal stromal tumors (GIST) with FDG PET, CT and MRI: a systematic review. Clin Transl Imaging 2017;5:183197.

33 Menge F, Jakob J, Kasper B, Smakic A, Marx A, Hohenberger P: Clinical presentation of gastrointestinal stromal tumors. Visc Med 2018;DOI: 10.1159/000494303.

34 Aghdassi A, Christoph A, Dombrowski F, et al: Gastrointestinal stromal tumors: clinical symptoms, location, metastasis formation, and associated malignancies in a single center retrospective study. Dig Dis 2018;36:337-345.

35 Marano L, Arru GM, Piras M, Fiume S, Gemini S: Surgical management of acutely presenting gastrointestinal stromal tumors of the stomach among elderly: experience of an emergency surgery department. Int J Surg 2014;12(suppl 1):S145-147.

36 Hecker A, Hecker B, Bassaly B, et al: Dramatic regression and bleeding of a duodenal GIST during preoperative imatinib therapy: case report and review. World J Surg Oncol 2010;8:47.

37 Eisenberg BL, Harris J, Blanke CD, et al: Phase II trial of neoadjuvant/adjuvant imatinib mesylate (IM) for advanced primary and metastatic/recurrent operable gastrointestinal stromal tumor (GIST): early results of RTOG 0132/ACRIN 6665. J Surg Oncol 2009;99:4247.

38 Doyon C, Sideris L, Leblanc G, Leclerc YE, Boudreau D, Dube P: Prolonged therapy with imatinib mesylate before surgery for advanced gastrointestinal stromal tumor results of a phase II trial. Int J Surg Oncol 2012; 2012:761576.

39 Kurokawa Y, Yang HK, Cho H, et al: Phase II study of neoadjuvant imatinib in large gastrointestinal stromal tumours of the stomach. Br J Cancer 2017;117:25-32.

40 Hohenberger P, Langer C, Wendtner CM, et al: Neoadjuvant treatment of locally advanced GIST: results of APOLLON, a prospective, open label phase II study in KIT- or PDGFRA-positive tumors. J Clin Oncol 2012; 30(suppl):10031.

41 Rutkowski P, Gronchi A, Hohenberger P, et al: Neoad juvant imatinib in locally advanced gastrointestinal stromal tumors (GIST): the EORTC STBSG experience. Ann Surg Oncol 2013;20:2937-2943.
42 Heinrich MC, Rankin C, Blanke CD, et al: Correlation of long-term results of imatinib in advanced gastrointestinal stromal tumors with next-generation sequencing results: analysis of phase 3 SWOG Intergroup Trial S0033. JAMA Oncol 2017;3:944-952.

43 Demetri GD, Reichardt P, Kang YK, et al: Efficacy and safety of regorafenib for advanced gastrointestinal stromal tumours after failure of imatinib and sunitinib (GRID): an international, multicentre, randomised, placebo-controlled, phase 3 trial. Lancet 2013;381: 295-302.

44 Demetri GD, van Oosterom AT, Garrett CR, et al: Efficacy and safety of sunitinib in patients with advanced gastrointestinal stromal tumour after failure of imatinib: a randomised controlled trial. Lancet 2006; 368:1329-1338.

45 Raut CP, Wang Q, Manola J, et al: Cytoreductive surgery in patients with metastatic gastrointestinal stromal tumor treated with sunitinib malate. Ann Surg Oncol 2010;17:407-415

46 Bose D, Meric-Bernstam F, Hofstetter W, Reardon DA, Flaherty KT, Ellis LM: Vascular endothelial growth factor targeted therapy in the perioperative setting: implications for patient care. Lancet Oncol 2010; 11:373-382.

47 Blay JY, Shen L, Kang YK, et al: Nilotinib versus imatinib as first-line therapy for patients with unresectable or metastatic gastrointestinal stromal tumours (ENESTg1): a randomised phase 3 trial. Lancet Oncol 2015;16:550-560.

48 Eriksson M, Reichardt P, Sundby Hall K, et al: Needle biopsy through the abdominal wall for the diagnosis of gastrointestinal stromal tumour - does it increase the risk for tumour cell seeding and recurrence? Eur J Cancer 2016;59:128-133.

49 Huss S, Elges S, Trautmann M, Sperveslage J, Hartmann W, Wardelmann E: Classification of KIT/PDGFRA wild-type gastrointestinal stromal tumors: implications for therapy. Expert Rev Anticancer Ther 2015; 15:623-628.

50 Le Cesne A, Van Glabbeke M, Verweij J, et al: Absence of progression as assessed by response evaluation criteria in solid tumors predicts survival in advanced GI stromal tumors treated with imatinib mesylate: the intergroup EORTC-ISG-AGITG phase III trial. J Clin Oncol 2009;27:3969-3974

51 Tirumani SH, Shinagare AB, Jagannathan JP, Krajewski KM, Ramaiya NH, Raut CP: Radiologic assessment of earliest, best, and plateau response of gastrointestinal stromal tumors to neoadjuvant imatinib prior to successful surgical resection. Eur J Surg Oncol 2014;40: 420-428.

52 Blesius A, Cassier PA, Bertucci F, et al: Neoadjuvant imatinib in patients with locally advanced non metastatic GIST in the prospective BFR14 trial. BMC Cancer 2011;11:72. 